\title{
Aortic enlargement in chronic obstructive pulmonary disease (COPD) and emphysema: The Multi-Ethnic Study of Atherosclerosis (MESA) COPD study尔
}

Kana Fujikura ${ }^{\mathrm{a}}$, Alessandra Albini ${ }^{\mathrm{b}}$, R. Graham Barr ${ }^{\mathrm{c}}$, Megha Parikh ${ }^{\mathrm{c}}$, Julia Kern ${ }^{\mathrm{c}}$, Eric Hoffman ${ }^{\mathrm{d}}$, Grant T. Hiura ${ }^{c}$, David A. Bluemke ${ }^{\mathrm{e}}$, James Carr ${ }^{\mathrm{f}}$, João A.C. Lima ${ }^{\mathrm{g}}$, Erin D. Michos ${ }^{\mathrm{g}}$, Antoinette S. Gomes ${ }^{\mathrm{h}}$, Martin R. Prince ${ }^{\mathrm{i}, *}$

a Advanced Cardiovascular Imaging Laboratory, National Heart, Lung and Blood Institute, National Institutes of Health, Department of Health and Human Services, Bethesda, ML, USA

b Department of Radiology, Columbia University, New York, USA

${ }^{c}$ Department of Medicine, Columbia University, New York, USA

d Department of Radiology, Medicine and Biomedical Engineering, University of Iowa, Iowa City, USA

e Department of Radiology, University of Wisconsin, Madison, USA

${ }^{\mathrm{f}}$ Department of Radiology, Northwestern University, Chicago, USA

${ }^{g}$ Division of Cardiology, Johns Hopkins University, Baltimore, USA

h Department of Radiology, University of California-Los Angeles, School of Medicine, Los Angeles, USA

i Department of Radiology, Weill Cornell Medicine, NY, New York, USA

\section{A R T I C L E I N F O}

\section{Article history:}

Received 12 April 2020

Received in revised form 25 December 2020

Accepted 5 February 2021

Available online 13 February 2021

\section{Keywords:}

Aortopathy

Aneurysm

MRI

MRA

Emphysema

COPD

\begin{abstract}
A B S T R A C T
Background: The prevalence of abdominal aortic aneurysm is high in chronic obstructive pulmonary disease (COPD) population. Emphysema involves proteolytic destruction of elastic fibers. Therefore, emphysema may also contribute to thoracic aorta dilatation. This study assessed aorta dilation in smokers stratified by presence of COPD, emphysema and airway thickening.

Methods: Aorta diameters were measured on 3D magnetic resonance angiography in smokers recruited from the Multi-Ethnic Study of Atherosclerosis (MESA), the Emphysema and Cancer Action Project (EMCAP), and the local community. COPD was defined by standard spirometric criteria; emphysema was measured quantitatively on computed tomography and bronchitis was determined from medical history.

Results: Participants ( $n=315$, age 58-79) included 150 with COPD and 165 without COPD, of whom 56\% and $19 \%$, respectively, had emphysema. Subjects in the most severe quartile of emphysematous change showed the largest diameter at all four aorta locations compared to those in the least severe quartiles (all $p<0.001$ ). Comparing subjects with and without COPD, aorta diameters were larger in participants with severe COPD in ascending and arch (both $\mathrm{p}<0.001)$, and abdominal aorta $(p=0.001)$. Chronic bronchitis and bronchial wall thickness did not correlate with aorta diameter. In subjects with emphysema, subjects with coexistence of COPD showed larger aorta than those without COPD in ascending $(p=0.003)$, arch $(p=0.002)$, and abdominal aorta $(p=0.04)$.

Conclusions: This study showed larger aorta diameter in subjects with COPD and severe emphysema compared to COPD related to chronic bronchitis or bronchial wall thickening.
\end{abstract}

(c) 2021 Elsevier B.V. All rights reserved.

Abbreviations: AAA, abdominal aortic aneurysm; ACC/AHA, American College of Cardiology/American Heart Association; ACE, Angiotensin-converting enzyme; ARB, Angiotensin II re-

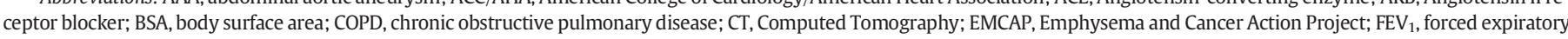

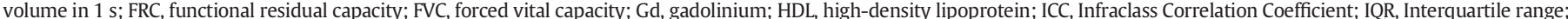

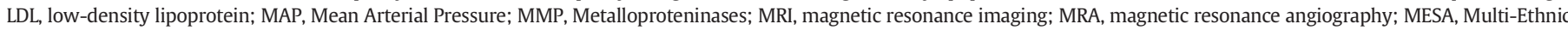

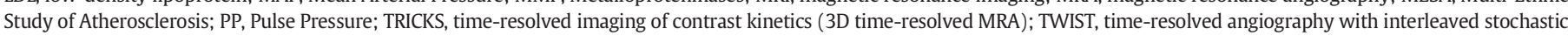
trajectories (3D time-resolved MRA).

t3 This author takes responsibility for all aspects of the reliability and freedom, from bias of the data presented and their discussed interpretation.

* Corresponding author at: 416 East 55th Street, New York, NY 10022, USA.

E-mail address: map2008@med.cornell.edu (M.R. Prince). 


\section{Introduction}

Advanced age and smoking are risk factors for aortic aneurysms as they are for other cardiovascular and pulmonary diseases [1-7]. Other known risk factors include increased pressure [8], i.e. hypertension or bicuspid aortic valve jets [1] and diseases that weaken the aortic wall including atherosclerosis [8], Marfan's and other connective tissue diseases [10].

High prevalence of aortic aneurysm is also reported in chronic obstructive pulmonary disease (COPD) [11] which is commonly observed in smokers. COPD is defined by airflow limitation on spirometry that does not fully reverse $[12,13]$. Smoking may also be associated with pulmonary emphysema, chronic bronchitis and airway thickening which can co-exist with COPD making it difficult to determine which features are more likely to be associated with aortic aneurysms [14]. This gap in knowledge is particularly relevant since emphysema and airway thickening can be measured on chest computed tomography (CT). Understanding which features are associated with aortic aneurysms could help potentially guide future screening and prevention strategies.

A striking histological feature of aortic aneurysms is destruction of the media and elastic tissue from excessive proteolytic enzyme activity in the aortic wall [2]. Emphysema also involves proteolytic destruction of elastic fibers. Thus, we hypothesize that both COPD and emphysema are at increased risk of aneurysm along the entire aorta. To test this hypothesis, we assessed aorta diameters in smokers stratified by presence of COPD, emphysema, chronic bronchitis and measures of airway thickening.

\section{Methods}

\subsection{Study participants}

The Multi-Ethnic Study of Atherosclerosis (MESA) is a prospective cohort study that recruited 6814 participants in 2000 to 2002 from six U.S. communities, who were white, African-American, Hispanic, or Chinese-American, aged 45-84 years and free of clinical cardiovascular disease. Between 2010 and 2012, 4716 participants returned for follow-up.

The MESA Lung Study enrolled 3965 MESA participants who all underwent full-lung CT and spirometry in 2010-2012.

The protocols of MESA and all studies described herein were approved by the Institutional Review Boards of all collaborating institutions (Columbia University, New York, NY; Johns Hopkins University, Baltimore, MD; Northwestern University, Chicago, IL; University of California, Los Angeles, LA; University of Minnesota, Twin Cities, MN; University of Iowa, Iowa City, IA) [15].

The population of this study was created from three cohorts: 1) all the subjects with COPD or emphysema from the MESA Lung Study $(n=190)$ [16], 2) all participants from the Emphysema and Cancer Action Project (EMCAP), a nonoverlapping lung cancer screening study $(n=89)$ [17], and 3) outpatient community at Columbia University Medical Center $(n=36)$, as described in previous studies [18,19]. Inclusion criteria were $50-79$ years of age with $\geq 10$ pack-year smoking history. Exclusion criteria were clinical cardiovascular disease (myocardial infarction, angina, heart failure, valve disease, atrial fibrillation or stroke), stage IIIb-V chronic kidney disease, asthma prior to age 45 years, prior lung resection, cancer, allergy to gadolinium, claustrophobia, metal in the body, pregnancy, and weight $>300 \mathrm{lbs}$. The present report includes these 315 participants who all underwent spirometry, CT scanning and contrast-enhanced cardiopulmonary magnetic resonance imaging (MRI) including three dimensional (3D) time-resolved magnetic resonance angiography (MRA, TRICKS or TWIST).

\subsubsection{Study oversight}

Study procedures were approved by institutional review boards of the participating institutions, Columbia University Medical Center (AAAD6395), Johns Hopkins University (NA_00030361/CR00017844),
Northwestern University (STU00021057-MOD0031), University of California, Los Angeles (11-002392-AM-00051), University of Washington (STUDY00001485), and by the National Heart, Lung, and Blood Institute. Written informed consent was obtained from all participants.

\subsection{Magnetic resonance imaging}

The MRI protocol included time-resolved 3D MRA to assess pulmonary vasculature [18]. Images were obtained using a 1.5 Tesla wholebody MRI system (Signa LX, GE Healthcare or Avanto, Siemens). TRICKS or TWIST was performed at $1.5 \mathrm{~s}$ temporal resolution following injection of $0.1 \mathrm{mMol} / \mathrm{kg}$ of Gd (Gd:DTPA, Bayer, Wayne, NY), and coronal 3D image of the aorta was obtained from cranial to aortic arch and caudal to aortic bifurcation.

\subsubsection{Aorta assessment}

MRA images were evaluated for the diameter of ascending aorta at the level of pulmonary artery, aortic arch at the level of brachiocephalic artery, intrathoracic descending aorta at the level of pulmonary artery, the abdominal aorta at the level of the mesenteric artery, and the infrarenal aorta immediately distal to the renal arteries. Aorta diameters were measured independently by a radiologist and a cardiologist blinded to all clinical information about the subjects using electronic calipers on a computer workstation with 3D visualization software (volume Viewer Plus Suite 15.10.4 on Advantage Windows Workstation (GE Medical Systems, Waukesha, WI). Reliability and interobserver agreement between the two readers were assessed by intraclass correlation coefficient (ICC). Discrepancies between the observers were resolved with a secondary consensus reading.

\subsection{COPD case status}

We used the standard definition of COPD to define case status: a post-bronchodilator ratio of the forced expiratory volume in one second $\left(\mathrm{FEV}_{1}\right)$ to the forced vital capacity $(\mathrm{FVC})$ ratio $<0.70[13,20]$ (Supplemental Fig. 1). Post-bronchodilator spirometry was measured in all participants based on American Thoracic Society/European Respiratory Society guidelines. Rolling barrel spirometer was used as previously described in the MESA Lung protocol [21]. COPD severity was classified as mild, FEV1 $\geq 80 \%$ predicted; moderate, FEV1 $50 \%$ to $79 \%$ predicted; and severe, FEV1 <50\% predicted [22].

\subsection{Computed tomography (CT), assessment of emphysema and airway anatomy}

All participants underwent full-lung CT scans with breath holding at maximum inspiration without intravenous contrast and reconstructed using a high-spatial-contrast algorithm with 0.625-mm-slice thickness on Siemens and GE 64-slice scanners (GE Healthcare, Waukesha, WI). All scans were acquired following the SPIROMICS protocol [16,17,23,31].

Image attenuation was assessed using a modified version of the Pulmonary Analysis Software Suite (VIDA Diagnostics, Coralville, IA) at a single reading center by trained readers without knowledge of other participants' information. Emphysema-like lung (also known as percent low attenuation area and hereafter referred to as percent emphysema) was defined as the number of lung voxels with outside-air corrected attenuation less than -950 Hounsfield units on chest CT [24] (Supplemental Table 1).

Stratified analyses defined patients with emphysema as those with percent emphysema voxels above the median value in this study. Sensitivity analyses used alternate thresholds of 25 th percentile, as well as median of emphysema [25].

The central airway tree was identified using Apollo Software (VIDA Diagnostics, Coralville, IA). Segmentation and labelling of the airways were visually verified by an image analyst and all labelled airways were assigned a generation number based upon the number of branch 
points from the trachea. Cross-sectional airway wall thickness and lumen area were measured at the 5 th generation perpendicular to the local airway segment's long axis and measurements were averaged along the middle third of each labelled airway segment [26].

Chronic bronchitis was defined as a chronic productive cough for 3 or more months in two or more years [21].

\subsection{Anthropometry, smoking status and other covarieties}

Age, gender, race/ethnicity, educational attainment, smoking status, pack-years, and medical history were self-reported. Medication use was assessed by medication inventory. Height and weight were measured following the MESA protocol [3]. Resting blood pressure was measured 3 times in the seated position. Glucose, total cholesterol and highdensity lipoprotein (HDL) cholesterol levels, and complete blood counts were measured from blood samples after at least twelve hour of fasting. Low-density lipoprotein (LDL) cholesterol level was calculated using the Friedewald equation [27]. Smoking history was assessed using standard questionnaire items and was confirmed with plasma cotinine levels [28]. Study participants reporting at least 1 cigarette in the 30 days prior to assessment were classified as current smokers. Information on medication use was obtained by medication inventory. Diabetes was defined as a fasting plasma glucose $\geq 126 \mathrm{mg} / \mathrm{dL}$ or self-report of physician diagnosis. Hypertension was defined as systolic blood pressure $\left(P_{\text {sys }}\right) \geq 140 \mathrm{mmHg}$, diastolic blood pressure $\left(\mathrm{P}_{\text {diast }}\right) \geq 90 \mathrm{mmHg}$ or physician diagnosis. The Mean Arterial Pressure (MAP) was calculated using the systolic and diastolic pressure values in the equation $\left(\mathrm{P}_{\text {diast }}+1 / 3\left(\mathrm{P}_{\text {sys }}-\mathrm{P}_{\text {dias }}\right)\right)$, and the Pulse Pressure (PP) as the difference between the systolic and diastolic pressure readings. All the blood pressure associated values were measured in $\mathrm{mmHg}[29]$.

\subsection{Statistical analysis}

Dichotomous variables are presented as proportions and continuous variables as means with standard deviation unless otherwise indicated. Fisher's exact test was used to assess the significance of differences in the number of aortic aneurysms based upon in percent emphysema and COPD severity. Multiple comparisons across levels of COPD severity were addressed by Holm's step-down procedure. Associations of aorta diameter with percent emphysema, COPD status and severity, chronic bronchitis status, and airway anatomy (wall thickness and inner area) were tested with linear regression. Potential confounders were selected based upon biological plausibility and examination of correlations with covariates. Unadjusted and adjusted linear regression models were performed, controlling for age, gender, race/ethnicity, height, weight, body surface area (BSA), cohort, smoking status, blood pressure, LDL-Cholesterol, HDL-Cholesterol and triglycerides, diabetes, smoking status, cotinine levels, and packyears smoked [30]. Given that study participants were recruited based

Table 1

Characteristics of the study participants by quartile of percent emphysema.

\begin{tabular}{|c|c|c|c|c|}
\hline & \multicolumn{4}{|c|}{ Percent emphysema ( $-950 \mathrm{HU})$} \\
\hline & $\begin{array}{l}\text { 1st Quartile } \\
\mathrm{n}=79\end{array}$ & $\begin{array}{l}\text { 2nd Quartile } \\
\mathrm{n}=79\end{array}$ & $\begin{array}{l}\text { 3rd Quartile } \\
\mathrm{n}=79\end{array}$ & $\begin{array}{l}\text { 4th Quartile } \\
\mathrm{n}=78\end{array}$ \\
\hline \multicolumn{5}{|l|}{ Demographics } \\
\hline Age, mean $\pm S D$, years & $66 \pm 7$ & $69 \pm 6$ & $68 \pm 7$ & $69 \pm 7$ \\
\hline Male, N (\%) & $36(46)$ & $44(56)$ & $53(67)$ & $55(70)$ \\
\hline \multicolumn{5}{|l|}{ Race/Ethnicity, N (\%) } \\
\hline White & $34(43)$ & $39(49)$ & $46(58)$ & $50(64)$ \\
\hline Chinese & $7(9)$ & $3(4)$ & $6(8)$ & $3(4)$ \\
\hline African-American & $24(30)$ & $22(28)$ & $17(21)$ & $20(26)$ \\
\hline Hispanic & $14(18)$ & $15(19)$ & $10(13)$ & $5(6)$ \\
\hline \multicolumn{5}{|l|}{ Educational attainment, N (\%) } \\
\hline$\leq$ High school degree & $24(30)$ & $12(24)$ & $16(20)$ & $17(22)$ \\
\hline Some college/Assoc. degree/Vocational school & $25(32)$ & $20(25)$ & $27(34)$ & $20(26)$ \\
\hline$\geq$ College degree & $30(38)$ & $40(51)$ & $36(45)$ & $41(52)$ \\
\hline Hypertension, N (\%) & $36(46)$ & $33(42)$ & $40(51)$ & $36(46)$ \\
\hline Diabetes Mellitus, N (\%) & $12(15)$ & $12(15)$ & $11(14)$ & $10(13)$ \\
\hline \multicolumn{5}{|l|}{ Smoking status, N (\%) } \\
\hline Current & $29(37)$ & $19(24)$ & $19(24)$ & $21(27)$ \\
\hline Former & $50(63)$ & $60(76)$ & $60(76)$ & $57(73)$ \\
\hline Pack-years of smoking, median [IQR] & $32[20-46]$ & $32[22-48]$ & $32[19-51]$ & $33[20-52]$ \\
\hline \multicolumn{5}{|l|}{ Characteristics } \\
\hline Height, mean $\pm \mathrm{SD}, \mathrm{cm}$ & $165 \pm 10$ & $168 \pm 10$ & $170 \pm 8$ & $171 \pm 9$ \\
\hline Weight, mean $\pm \mathrm{SD}, \mathrm{kg}$ & $78 \pm 17$ & $83 \pm 18$ & $80 \pm 16$ & $78 \pm 18$ \\
\hline Body mass index, mean $\pm \mathrm{SD}, \mathrm{kg} / \mathrm{m}^{2}$ & $28 \pm 5$ & $29 \pm 5$ & $28 \pm 5$ & $27 \pm 5$ \\
\hline Body surface area, mean $\pm S D, \mathrm{~m}^{2}$ & $1.8 \pm 0.22$ & $1.9 \pm 0.23$ & $1.9 \pm 0.20$ & $1.9 \pm 0.23$ \\
\hline Systolic blood pressure, mean $\pm \mathrm{SD}, \mathrm{mmHg}$ & $122 \pm 17$ & $121 \pm 18$ & $121 \pm 20$ & $122 \pm 15$ \\
\hline Diastolic blood pressure, mean $\pm \mathrm{SD}, \mathrm{mmHg}$ & $70 \pm 10$ & $69 \pm 9$ & $69 \pm 10$ & $72 \pm 8$ \\
\hline $\mathrm{MAP}$, mean $\pm \mathrm{SD}, \mathrm{mmHg}$ & $87 \pm 11$ & $86 \pm 10$ & $87 \pm 12$ & $89 \pm 9$ \\
\hline $\mathrm{PP}$, mean $\pm \mathrm{SD}, \mathrm{mmHg}$ & $51 \pm 15$ & $52 \pm 15$ & $52 \pm 15$ & $49 \pm 13$ \\
\hline \multicolumn{5}{|l|}{ Labs } \\
\hline Cotinine levels, mean $\pm \mathrm{SD}$, mg/dL & $133 \pm 211$ & $360 \pm 2245$ & $88 \pm 180$ & $139 \pm 647$ \\
\hline $\mathrm{LDL}$, mean $\pm \mathrm{SD}, \mathrm{mg} / \mathrm{dL}$ & $106 \pm 31$ & $109 \pm 32$ & $110 \pm 32$ & $97 \pm 31$ \\
\hline $\mathrm{HDL}$, mean $\pm \mathrm{SD}, \mathrm{mg} / \mathrm{dL}$ & $58 \pm 20$ & $54 \pm 16$ & $58 \pm 19$ & $56 \pm 18$ \\
\hline Triglycerides, mean $\pm \mathrm{SD}, \mathrm{mg} / \mathrm{dL}$ & $101 \pm 44$ & $112 \pm 50$ & $109 \pm 43$ & $120 \pm 84$ \\
\hline Cholesterol, median [IQR] & $178[160-205]$ & $182[159-210]$ & $187[160-222]$ & $178[147-196]$ \\
\hline \multicolumn{5}{|l|}{ Pulmonary function test } \\
\hline $\mathrm{FEV}_{1}$ percent of predicted, mean $\pm \mathrm{SD}$ & $91 \pm 17$ & $95 \pm 20$ & $92 \pm 17$ & $75 \pm 25$ \\
\hline FVC percent of predicted, mean \pm SD & $92 \pm 13$ & $97 \pm 14$ & $101 \pm 16$ & $97 \pm 18$ \\
\hline $\mathrm{FEV}_{1} / \mathrm{FVC}$ ratio, mean $\pm \mathrm{SD}$ & $0.75 \pm 0.09$ & $0.73 \pm 0.08$ & $0.69 \pm 0.1$ & $0.56 \pm 0.14$ \\
\hline
\end{tabular}

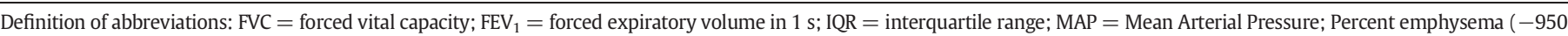
$\mathrm{HU})=$ percentage of emphysema-like lung at -950 Hounsfield units; PP = Pulse Pressure. 
upon COPD status rather than as a cohort study, analyses of percent emphysema and other continuous variables were weighted proportionate to the inverse ratio of the sample prevalence to the source study prevalence, COPD cases recruited from the community were assigned the same weights as cases from EMCAP [31]. A two-tailed $P$ value $<0.05$ was considered statistically significant. Analyses were performed using SAS 9.4 (Cary, NC) statistical software.

\section{Results}

The 315 participants had a mean age of $68 \pm 7$ years old, and 188 (60\%) were male. Race/ethnic distribution was 169 (54\%) White, 83 (26\%) African-American, 44 (14\%) Hispanic, and 19 (6\%) Asian. All participants were either current $(n=88)$ or former $(n=227)$ smokers. Additional demographic data are presented in Table 1.

Intra- and inter-observer agreements of the aorta diameter measurements are as follows: ascending aorta (ICC $=0.71,0.89$, respectively), arch (ICC $=0.72,0.74$, respectively), descending aorta (ICC $=$ $0.75,0.79$, respectively), abdominal aorta (ICC $=0.77,0.86$, respectively), infra-renal aorta (ICC $=0.76,0.83$, respectively).

\subsection{Aorta diameter and emphysema}

Ascending, arch, descending, abdominal and infra-renal aorta diameters were monotonically related to percent emphysema in unadjusted analyses (Table $2 \mathrm{~A}$; all $P<0.001$ ). This positive association of aortic diameter with percent emphysema was statistically significant in the fully adjusted multivariable model (Table 2B, Fig. 1A).

Using $\geq 40 \mathrm{~mm}$ as the threshold for diagnosing ascending aortic aneurysm, and $\geq 30 \mathrm{~mm}$ for diagnosing abdominal aneurysm, 30 of the 315 participants had aneurysm, with a maximum diameter $=48 \mathrm{~mm}$. There were 11 aneurysms in the 4th quartile of emphysema severity, 5 in the 3rd quartile, and 11 in the 2nd quartile compared to 3 in the 1 st quartile participants. Therefore, in subjects with emphysema, the number of aneurysms increased across quartiles ( $p=0.04$ ) of emphysema severity (Fig. 1B).

\subsection{Aorta diameter and COPD severity}

Greater severity of COPD was associated with greater diameter of the ascending aorta, aortic arch, and abdominal aorta (all $p$-values for trends across COPD severities categories $<0.001$ ), with little changes after multivariable adjustment (Table 3 ). Diameters of the aortic arch and abdominal aorta were significantly greater in severe, moderate and mild COPD compared to participants with no COPD; the ascending aorta diameter was significantly greater in severe and moderate COPD compared to no COPD; and the descending thoracic aorta was significantly in greater in diameter in severe COPD only. Although aortic diameter was greater with COPD, this did not translate into more aortic aneurysms. Among the 30 total aneurysms, 11 were among the 154 participants without COPD, 8 were among the 53 participants with mild COPD, 8 were among the 62 subjects with moderate COPD, and 3 were among the 13 individuals with severe COPD, showing no linear relationship $(p=0.2)$.

\subsection{Aorta diameter and coexistence of emphysema and COPD}

In subjects with emphysema, those with coexistence of COPD showed greater aorta diameter than those without COPD in ascending $(p=0.003)$, arch $(p=0.002)$, and abdominal aorta $(p=0.04)$ (Supplemental Table 1).

\subsection{Aorta diameter and chronic bronchitis}

Both the unadjusted and adjusted models showed no evidence for a continuous association of chronic bronchitis with greater aortic diameter (Supplemental Table 2).

Table 2

Aorta diameters ( $\mathrm{mm}$ ) according to emphysema quartiles.

\begin{tabular}{|c|c|c|c|c|c|}
\hline & \multicolumn{4}{|c|}{ Percent of emphysema-like lung } & \multirow[b]{2}{*}{$p$-value } \\
\hline & $\begin{array}{l}\text { 1st Quartile } \\
(\mathrm{n}=79) \\
\text { Normal } \\
\text { Mean } \pm \mathrm{SD}\end{array}$ & $\begin{array}{l}\text { 2nd Quartile } \\
(\mathrm{n}=79) \\
\text { Borderline } \\
\text { Mean } \pm \text { SD }\end{array}$ & $\begin{array}{l}\text { 3rd Quartile } \\
(\mathrm{n}=79) \\
\text { Mild } \\
\text { Mean } \pm \text { SD }\end{array}$ & $\begin{array}{l}\text { 4th Quartile } \\
(\mathrm{n}=78) \\
\text { Severe } \\
\text { Mean } \pm \mathrm{SD}\end{array}$ & \\
\hline Ascending Aorta & $30 \pm 3$ & $31 \pm 4$ & $34 \pm 3$ & $35 \pm 4$ & $<0.001$ \\
\hline Arch & $26 \pm 2$ & $27 \pm 2$ & $28 \pm 3$ & $29 \pm 3$ & $<0.001$ \\
\hline Descending Aorta & $23 \pm 3$ & $24 \pm 3$ & $27 \pm 3$ & $27 \pm 3$ & $<0.001$ \\
\hline Abdominal Aorta & $20 \pm 3$ & $21 \pm 3$ & $23 \pm 3$ & $24 \pm 3$ & $<0.001$ \\
\hline Infrarenal Aorta & $14 \pm 3$ & $14 \pm 3$ & $16 \pm 2$ & $17 \pm 3$ & $<0.001$ \\
\hline \multicolumn{6}{|l|}{ (B) Adjusted model. } \\
\hline & \multicolumn{4}{|c|}{ Percent of emphysema-like lung } & \multirow[b]{2}{*}{ p-value } \\
\hline & $\begin{array}{l}\text { 1st Quartile } \\
(\mathrm{n}=79) \\
\text { Normal }\end{array}$ & $\begin{array}{l}\text { 2nd Quartile } \\
(\mathrm{n}=79) \\
\text { Borderline } \\
\beta(95 \% \mathrm{CI})\end{array}$ & $\begin{array}{l}\text { 3rd Quartile } \\
(\mathrm{n}=79) \\
\text { Mild } \\
\beta(95 \% \mathrm{CI})\end{array}$ & $\begin{array}{l}\text { 4th Quartile } \\
(\mathrm{n}=77) \\
\text { Severe } \\
\beta(95 \% \mathrm{CI})\end{array}$ & \\
\hline Ascending Aorta & Ref & $0.71(-0.29,1.71)$ & $3.25(2.26,4.24)$ & $4.19(2.98,5.41)$ & $<0.001$ \\
\hline Arch & Ref & $0.59(-0.18,1.35)$ & $1.84(1.05,2.64)$ & $2.32(1.37,3.26)$ & $<0.001$ \\
\hline Descending Aorta & Ref & $0.74(-0.05,1.53)$ & $2.80(2.05,3.54)$ & $2.86(2.07,3.65)$ & $<0.001$ \\
\hline Abdominal Aorta & Ref & $0.06(-0.74,0.87)$ & $2.82(2.00,3.64)$ & $2.69(1.84,3.54)$ & $<0.001$ \\
\hline Infrarenal Aorta & Ref & $-0.47(-1.40,0.46)$ & $1.77(0.86,2.68)$ & $2.57(1.58,3.57)$ & $<0.001$ \\
\hline
\end{tabular}

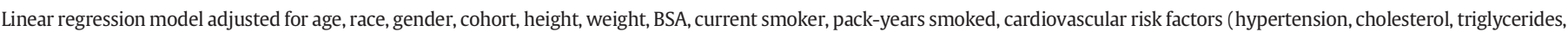
LDL and diabetes). 

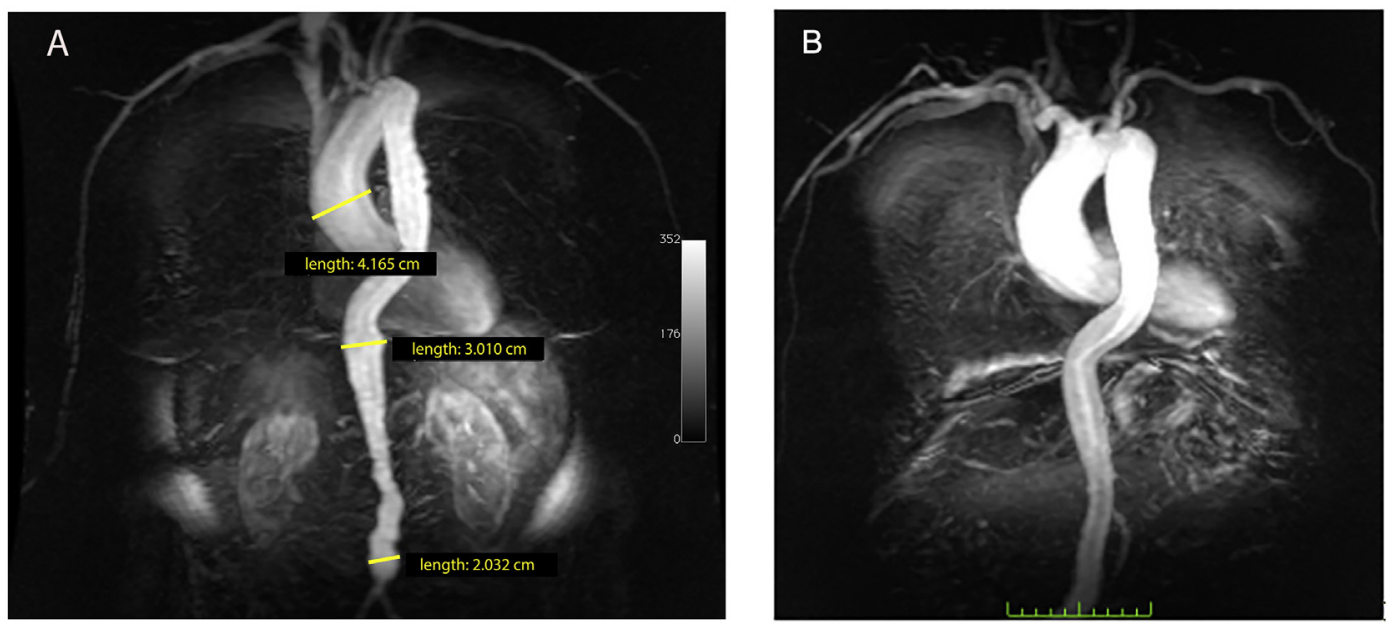

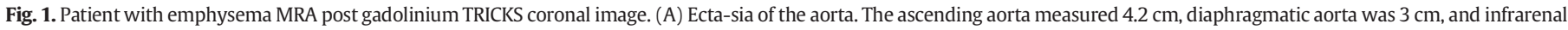
aorta $2 \mathrm{~cm}$ ectasia. (B) Aneurysmal ascending aorta.

\subsection{Aorta diameter and airway anatomy}

Bronchial wall thickness was not associated with any difference in aorta diameter with the fully adjusted model (Supplemental Table 3). Furthermore, there was no evidence for a continuous association of bronchial lumen area and aorta diameter (Supplemental Table 4).

\section{Discussion}

This large and ethnically diverse population allowed for multiple stratified analyses of aortic aneurysm risk in COPD according to the presence of emphysema, chronic bronchitis and bronchial wall thickening. Among 315 current and former smokers, subjects with COPD and especially emphysema, diagnosed on CT, showed greater thoracic and abdominal aorta cross-sectional diameters, compared to controls with no COPD. A greater number of subjects with severe emphysema showed thoracic and abdominal aortic aneurysms compared to participants without emphysema. Increased aorta diameter was not correlated with chronic bronchitis or airway dimensions.

Aortic aneurysm is the result of multifactorial processes, including inflammation, genetic abnormalities, biomechanical wall stress, apoptosis, and proteolytic degradation of connective tissue including elastin and collagen [32,33]. Risk factors for development of aortic aneurysms include factors that increase stress on the aortic wall (e.g. hypertension and elevated diastolic blood pressure), factors that promote degradation of the elastin and collagen fibers within the aorta wall (e.g. aging, smoking, atherosclerosis), and genetic factors that predispose the aortic wall to degeneration (e.g. Marfan syndrome, Ehler-Danlos, Cutis Laxa).

Since emphysema also results from destruction of elastin and collagen $[34,35]$, it is not surprising that emphysema is associated with

Table 3

Aorta diameters ( $\mathrm{mm}$ ) across COPD severity groups.

(A) Distributions of aorta diameters, by COPD severity.

\begin{tabular}{|c|c|c|c|c|c|}
\hline & \multirow[b]{2}{*}{$\begin{array}{l}\text { Normal } \\
(\mathrm{n}=165) \\
\text { Mean } \pm \mathrm{SD}\end{array}$} & \multicolumn{3}{|c|}{ COPD on spirometry } & \multirow[b]{2}{*}{$\mathrm{p}$-value for trend } \\
\hline & & $\begin{array}{l}\text { Mild } \\
(n=61) \\
\text { Mean } \pm S D\end{array}$ & $\begin{array}{l}\text { Moderate } \\
(\mathrm{n}=70) \\
\text { Mean } \pm \mathrm{SD}\end{array}$ & $\begin{array}{l}\text { Severe } \\
(n=19) \\
\text { Mean } \pm S D\end{array}$ & \\
\hline Ascending Aorta, & $32 \pm 4$ & $33 \pm 4$ & $33 \pm 5$ & $34 \pm 4$ & $<0.001$ \\
\hline Arch & $27 \pm 3$ & $28 \pm 3$ & $28 \pm 3$ & $29 \pm 3$ & $<0.001$ \\
\hline Descending Aorta & $24 \pm 3$ & $26 \pm 3$ & $25 \pm 4$ & $26 \pm 4$ & 0.11 \\
\hline Abdominal Aorta & $21 \pm 3$ & $22 \pm 3$ & $22 \pm 4$ & $24 \pm 3$ & $<0.001$ \\
\hline Infrarenal Aorta & $15 \pm 3$ & $16 \pm 2$ & $16 \pm 3$ & $17 \pm 3$ & $<0.001$ \\
\hline \multicolumn{6}{|l|}{ (B) Adjusted model } \\
\hline & & \multicolumn{3}{|l|}{ COPD on spirometry } & \multirow[b]{2}{*}{$\mathrm{p}$-value for trend } \\
\hline & $\begin{array}{l}\text { Normal } \\
(\mathrm{n}=164)\end{array}$ & $\begin{array}{l}\text { Mild } \\
(\mathrm{n}=61) \\
\beta(95 \% \mathrm{CI})\end{array}$ & $\begin{array}{l}\text { Moderate } \\
(\mathrm{n}=70) \\
\beta(95 \% \mathrm{CI})\end{array}$ & $\begin{array}{l}\text { Severe } \\
(\mathrm{n}=19) \\
\beta(95 \% \mathrm{Cl})\end{array}$ & \\
\hline Ascending Aorta, mm & Ref & $0.98(-0.16,2.12)$ & $1.60(0.50,2.71)^{*}$ & $2.80(0.74,4.86)^{*}$ & $<0.001$ \\
\hline Arch, mm & Ref & $1.06(0.25,1.87)^{*}$ & $1.09(0.29,1.89)^{*}$ & $2.32(0.85,3.78)^{*}$ & $<0.001$ \\
\hline Descending Aorta, mm & Ref & $0.74(-0.13,1.61)$ & $0.81(-0.04,1.65)$ & $1.80(0.23,3.37)^{*}$ & 0.02 \\
\hline Abdominal Aorta, mm & Ref & $1.11(0.22,1.99)^{*}$ & $1.14(0.24,2.03)^{*}$ & $2.32(0.72,3.91)^{*}$ & 0.001 \\
\hline Infrarenal Aorta, mm & Ref & $1.12(0.26,1.98)^{*}$ & $1.15(0.27,2.04)^{*}$ & $1.11(-0.42,2.65)$ & 0.01 \\
\hline
\end{tabular}

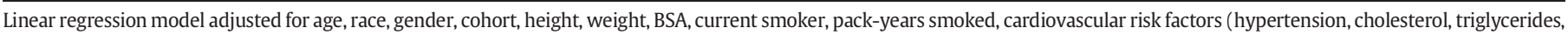
LDL and diabetes).

* $P$-value $<0.05$, indicating statistical significance compared to Normal. 
increasing aortic diameters and aortic aneurysms [36]. Tobacco smoke inhibits the anti-elastase activity of alpha1-antitrypsin (alpha1 - protease inhibitor), causing an imbalance between elastolytic and antielastolytic factors, which leads to emphysema [6]. Furthermore, autoimmune T-cell activation has been shown to be associated with smoking in patients with thoracic aortic aneurysm or dissection without COPD [37]. Destruction of elastin in aortic aneurysms may be via the same mechanism.

The relative risk of abdominal aortic aneurysms (AAA) in individuals who have ever smoked is 2.5 times greater than the relative risk for coronary heart disease [4]. Lederle et al. [5] described AAA more closely associated with cigarette smoking than any other tobacco-related disease except lung cancer. MacSweeney et al. [7] assessed active smoking (serum cotinine levels), blood pressure, cholesterol, and triglycerides in patients with small ( $<40 \mathrm{~mm}$ ) AAAs. Active smoking was the only parameter associated with a significant increase in growth rate, and double the rate of thoraco-abdominal aneurysm expansion [9]. In Marfan syndrome [10], subjects are highly predisposed to thoracic aortic aneurysm and/or Type A dissection, with virtually every patient developing aortic disease at some point during their lifetime [38]. In addition, giant bullous and emphysema have been reported in Marfan patients [39]. Cystic medial aortic degeneration also occurs in EhlersDanlos syndrome [40] and in particular the vascular type IV showed the presence of bullous emphysema and aortic aneurysm [41]. Individuals with Cutis Laxa (generalized elastolysis), characterized by abnormal elastic fibers and loose skin [43], also showed aortic aneurysm and emphysema [44].

Bicuspid aortic valve is often seen in patients with connective tissue diseases. The ACC/AHA Valvular Heart Disease Guidelines specifically address bicuspid aortic valve as a cause of thoraco-abdominal aneurysm [1], and there are reports of bicuspid aortic valve patients with emphysema [44]. There are also reports of aortic dissection in patients with autosomal dominant polycystic kidney disease [42]. Previous studies showed a coexistence of polycystic kidney disease and alpha-1 antitrypsin deficiency associated emphysema [45].

The continuous association between greater aortic diameters and emphysema may also relate to endothelial damage; endothelium is present in both airways and vessels [46]. Several proinflammatory cytokines, including interleukin-6, interleukin- $1 \beta$, tumor necrosis factor- $\alpha$, and interferon- $\gamma$, have been identified in AAA walls. These cytokines activate lymphocytes and macrophages [32] and promote matrix metalloproteinases MMP-2 and MMP-9 [47] production in the smooth muscle of aortic wall that degrade elastin and collagen and leads to the cystic medial necrosis found in aortic aneurysms [37]. These inflammatory processes are seen in both aneurysms and emphysema [33]. A recent study in animal models, has showed how treatment with angiotensinconverting enzyme (ACE) inhibitors and angiotensin II receptor blockers (ARBs), inhibit transforming growth factor- $\beta$ in the endothelium, improve endothelial function and restore airspace architecture. ARB/ACE inhibitor therapy was associated with slower progression of percent emphysema over ten years, particularly among former smokers. ACE and ARB agents are currently being developed for the prevention and treatment of emphysema [48]. ACE inhibitors are also used in preventing aortic aneurysm rupture [49].

We used validated instruments including MRA, Spirometry and CT with data collected in a blinded manner. MRA has an advantage that it does not risk patients with radiation. Especially for patients requiring series of imaging tests to follow-up disease processes, cumulative radiation dose via CT may become somewhat problematic. TRICKS or TWIST has reasonable temporal and spatial resolution for aortic diameter measurements and allows the visualization of whole aorta with optimal contrast-enhancement.

There are some limitations in this study. First, our study was observational and cross-sectional, thus neither causality nor temporality can be inferred. Secondly, there may be residual confounding factors that were not included in our models. The study includes a limited age range, 50-79 years. Aorta diameter had to be obtained from MRA instead of the higher resolution CT images since the CT was noncontrast and low dose optimized for assessing lung parenchyma in volunteers not aorta diamter.

\section{Conclusions}

In this large, multi-ethnic sample of individuals who are former/current smokers free of clinical cardiovascular disease recruited from the community, we have found that COPD with emphysema is associated with increased thoracic and abdominal aortic diameters but this is not observed in the chronic bronchitis and thickened airway subtypes of COPD. There may be similar elastolytic mechanisms destroying lung parenchyma to cause emphysema and weakening the aorta wall, however it requires further investigation.

Supplementary data to this article can be found online at https://doi. org/10.1016/j.ijcard.2021.02.017.

\section{Funding}

This research was supported by grants R01-HL093081, R01HL077612, and R01-HL121270 and contracts 75N92020D00001, HHSN268201500003I, N01-HC-95159, 75N92020D00005, N01-HC95160, 75N92020D00002, N01-HC-95161, 75N92020D00003, N01HC-95162, 75N92020D00006, N01-HC-95163, 75N92020D00004, N01-HC-95164, 75N92020D00007, N01-HC-95165, N01-HC-95166, N01-HC-95167, N01-HC-95168 and N01-HC-95169 from the National Heart, Lung, and Blood Institute (NHLBI), National Institutes of Health (NIH) and by grants UL1-TR-000040, UL1-TR-001079, and UL1-TR001420 from the National Center for Advancing Translational Sciences (NCATS). This work was partially supported by the Division of Intramural Research, NHLBI, NIH, Bethesda, MD, USA of Health and Human Services (DHHS).

\section{Declaration of Competing Interest}

None.

\section{Acknowledgments}

Authors would like to acknowledge Benjamin M. Smith ${ }^{1}$, MD, MS and Chia $\mathrm{Liu}^{2}$, PhD for their contribution to the conception and study design and in the acquisition, analysis and interpretation of the data.

\section{References}

[1] C.M. Otto, R.A. Nishimura, R.O. Bonow, et al., ACC/AHA guideline for the management of patients with valvular heart disease: a report of the American College of Cardiology/American Heart Association Joint Committee on Practice Guidelines, Circulation 2020 (2020) (online ahead of print).

[2] M.J. Davies, Aortic aneurysm formation: lessons from human studies and experimental models, Circulation 98 (1998) 193-195.

[3] J.T. Powell, P. Worrell, S.T. MacSweeney, P.J. Franks, R.M. Greenhalgh, Smoking as a risk factor for abdominal aortic aneurysm, Ann. N. Y. Acad. Sci. 800 (1996) 246-248.

[4] F.A. Lederle, D.B. Nelson, A.M. Joseph, Smokers' relative risk for aortic aneurysm compared with other smoking-related diseases: a systematic review, J. Vasc. Surg. 38 (2003) 329-334.

[5] T. Muley, M. Wiebel, V. Schulz, W. Ebert, Elastinolytic activity of alveolar macrophages in smoking-associated pulmonary emphysema, Clin Investig 72 (1994) 269-276.

[6] S.T. MacSweeney, M. Ellis, P.C. Worrell, R.M. Greenhalgh, J.T. Powell, Smoking and growth rate of small abdominal aortic aneurysms, Lancet 344 (1994) 651-652.

[7] E. Vizzardi, F. Maffessanti, R. Lorusso, et al., Ascending aortic dimensions in hypertensive subjects: reference values for two-dimensional echocardiography, J. Am. Soc. Echocardiogr. 29 (2016) 827-837.

[8] O.E. Dapunt, J.D. Galla, A.M. Sadeghi, et al., The natural history of thoracic aortic aneurysms, J. Thorac. Cardiovasc. Surg. 107 (1994) 1323-1332.

[9] R.W. Jeremy, H. Huang, J. Hwa, H. McCarron, C.F. Hughes, J.G. Richards, Relation between age, arterial distensibility, and aortic dilatation in the Marfan syndrome, Am. J. Cardiol. 74 (1994) 369-373. 
[10] Y. Kubota, A.R. Folsom, K. Matsushita, D. Couper, W. Tang, Prospective study of lung function and abdominal aortic aneurysm risk: the atherosclerosis risk in communities study, Atherosclerosis 268 (2018) 225-230.

[11] C.F. Vogelmeier, G.J. Criner, F.J. Martinez, et al., Global strategy for the diagnosis, management, and prevention of chronic obstructive lung disease 2017 report. GOLD Executive Summary, Am. J. Respir. Crit. Care Med. 195 (2017) 557-582.

[12] S.E. Marsh, J. Travers, M. Weatherall, et al., Proportional classifications of COPD phenotypes, Thorax 63 (2008) 761-767.

[13] Gold Reports, Global initiative for chronic obstructive lung disease, 2021 https:// goldcopd.org/2021-gold-reports/ (accessed 16 December 2020).

[14] C.P. Aaron, E.A. Hoffman, J.A.C. Lima, et al., Pulmonary vascular volume, impaired left ventricular filling and dyspnea: the MESA lung study, PLoS One 12 (2017), e0176180,

[15] D.E. Bild, D.A. Bluemke, G.L. Burke, et al., Multi-ethnic study of atherosclerosis: objectives and design, Am. J. Epidemiol. 156 (2002) 871-881.

[16] K. Hueper, J. Vogel-Claussen, M.A. Parikh, et al., Pulmonary microvascular blood flow in mild chronic obstructive pulmonary disease and emphysema. The MESA COPD study, Am. J. Respir. Crit. Care Med. 192 (2015) 570-580.

[17] R.G. Barr, S. Mesia-Vela, J.H. Austin, et al., Impaired flow-mediated dilation is associated with low pulmonary function and emphysema in ex-smokers: the emphysema and cancer action project (EMCAP) study, Am. J. Respir. Crit. Care Med. 176 (2007) 1200-1207.

[18] S.M. Kawut, H.D. Poor, M.A. Parikh, et al., Cor pulmonale parvus in chronic obstructive pulmonary disease and emphysema: the MESA COPD study, J. Am. Coll. Cardiol. 64 (2014) 2000-2009.

[19] J.L. Hankinson, J.R. Odencrantz, K.B. Fedan, Spirometric reference values from a sample of the general U.S. population, Am. J. Respir. Crit. Care Med. 159 (1999) 179-187.

[20] J.L. Hankinson, S.M. Kawut, E. Shahar, L.J. Smith, K.H. Stukovsky, R.G. Barr, Performance of American Thoracic Society-recommended spirometry reference values in a multiethnic sample of adults: the multi-ethnic study of atherosclerosis (MESA) lung study, Chest 137 (2010) 138-145.

[21] J.P. Sieren, J.D. Newell Jr., R.G. Barr, et al., SPIROMICS protocol for multicenter quantitative computed tomography to phenotype the lungs, Am. J. Respir. Crit. Care Med. 194 (2016) 794-806.

[22] B.R. Celli, W. MacNee, A. Agusti, et al., Standards for the diagnosis and treatment of patients with COPD: a summary of the ATS/ERS position paper, Eur. Respir. J. 23 (2004) 932-946.

[23] R.G. Barr, D.A. Bluemke, F.S. Ahmed, et al., Percent emphysema, airflow obstruction, and impaired left ventricular filling, N. Engl. J. Med. 362 (2010) 217-227.

[24] E.C. Oelsner, B.M. Smith, E.A. Hoffman, et al., Associations between emphysema-like lung on CT and incident airflow limitation: a general population-based cohort study, Thorax 73 (2018) 486-488.

[25] B.M. Smith, E.A. Hoffman, D. Rabinowitz, et al., Comparison of spatially matched airways reveals thinner airway walls in COPD. The multi-ethnic study of atherosclerosis (MESA) COPD study and the subpopulations and intermediate outcomes in COPD study (SPIROMICS), Thorax 69 (2014) 987-996.

[26] K.M. Burkart, A. Manichaikul, J.B. Wilk, et al., APOM and high-density lipoprotein cholesterol are associated with lung function and per cent emphysema, Eur. Respir. J. 43 (2014) 1003-1017.

[27] J. Rodriguez, R. Jiang, W.C. Johnson, B.A. MacKenzie, LJ. Smith, R.G. Barr, The association of pipe and cigar use with cotinine levels, lung function, and airflow obstruction: a cross-sectional study, Ann. Intern. Med. 152 (2010) 201-210.

[28] A.V. Chobanian, G.L. Bakris, H.R. Black, et al., The seventh report of the joint National Committee on prevention, detection, evaluation, and treatment of high blood pressure: the JNC 7 report, JAMA 289 (2003) 2560-2572.

[29] A.J. Podolanczuk, E.C. Oelsner, R.G. Barr, et al., High attenuation areas on chest computed tomography in community-dwelling adults: the MESA study, Eur. Respir. J. 48 (2016) 1442-1452.
[30] B.M. Smith, S.M. Kawut, D.A. Bluemke, et al., Pulmonary hyperinflation and left ventricular mass: the multi-ethnic study of atherosclerosis COPD study, Circulation 127 (2013) 1503-1511.

[31] J.M. Reilly, C.M. Brophy, M.D. Tilson, Characterization of an elastase from aneurysmal aorta which degrades intact aortic elastin, Ann. Vasc. Surg. 6 (1992) 499-502.

[32] J.D. Maclay, D.A. McAllister, R. Rabinovich, et al., Systemic elastin degradation in chronic obstructive pulmonary disease, Thorax 67 (2012) 606-612.

[33] L. Segura-Valdez, A. Pardo, M. Gaxiola, B.D. Uhal, C. Becerril, M. Selman, Upregulation of gelatinases A and B, collagenases 1 and 2, and increased parenchymal cell death in COPD, Chest 117 (2000) 684-694.

[34] Y. Kasahara, R.M. Tuder, C.D. Cool, N.F. Voelkel, Expression of 15-lipoxygenase and evidence for apoptosis in the lungs from patients with COPD, Chest 117 (2000) 260s.

[35] J.S. Lindholt, L. Heickendorff, S. Antonsen, H. Fasting, E.W. Henneberg Natural history of abdominal aortic aneurysm with and without coexisting chronic obstructive pulmonary disease, J. Vasc. Surg. 28 (1998) 226-233.

[36] B.H. Gu, J.C. Choi, Y.H. Shen, et al., Elastin-specific autoimmunity in smokers with thoracic aortic aneurysm and dissection is independent of chronic obstructive pulmonary disease, J. Am. Heart Assoc. 8 (2019), e011671,

[37] P. Meffert, A. Tscheuschler, F. Beyersdorf, et al., Characterization of serum matrix metalloproteinase 2/9 levels in patients with ascending aortic aneurysms, Interact. Cardiovasc. Thorac. Surg. 24 (2017) 20-26.

[38] A.G. Corsico, A. Grosso, B. Tripon, et al., Pulmonary involvement in patients with Marfan syndrome, Panminerva Med. 56 (2014) 177-182.

[39] V.R. Shannon, A.S. Nanda, S.A. Faiz, Marfan syndrome presenting as Giant bullous emphysema, Am. J. Respir. Crit. Care Med. 195 (2017) 827-828.

[40] R. Ascione, W.J. Gomes, M. Bates, J.L. Shannon, F.M. Pope, G.D. Angelini, Emergency repair of type a aortic dissection in type IV Ehlers-Danlos syndrome, Cardiovasc. Surg. 8 (2000) 75-78.

[41] M. Pepin, U. Schwarze, A. Superti-Furga, P.H. Byers, Clinical and genetic features of Ehlers-Danlos syndrome type IV, the vascular type, N. Engl. J. Med. 342 (2000) 673-680.

[42] D.R. Berk, D.D. Bentley, S.J. Bayliss, A. Lind, Z. Urban, Cutis laxa: a review, J. Am. Acad. Dermatol. 66 (2012) 842.e1-17.

[43] P. Champion, F. Ryan, A case of congenital cutis laxa (generalized elastolysis), Can. Respir. J. 12 (2005) 151-152.

[44] T. Carrel, P. Berdat, M. Pavlovic, S. Sukhanov, L. Englberger, J.P. Pfammatter, Surgery of the dilated aortic root and ascending aorta in pediatric patients: techniques and results, Eur. J. Cardiothorac. Surg. 24 (2003) 249-254.

[45] S. Pintacuda, S. Di Blasi, G. Morici, S. Amato, The polycystic kidney and serum alpha1-antitrypsin deficiency. Observations on 2 family groups, Minerva Med. 72 (1981) 1697-1701.

[46] M.R. Bersi, R. Khosravi, A.J. Wujciak, D.G. Harrison, J.D. Humphrey, Differential cellmatrix mechanoadaptations and inflammation drive regional propensities to aortic fibrosis, aneurysm or dissection in hypertension, J. R. Soc. Interface 14 (2017).

[47] A. Tscheuschler, P. Meffert, F. Beyersdorf, et al., MMP-2 isoforms in aortic tissue and serum of patients with ascending aortic aneurysms and aortic root aneurysms, PLoS One 11 (2016), e0164308, .

[48] M.A. Parikh, C.P. Aaron, E.A. Hoffman, et al., Angiotensin-converting inhibitors and angiotensin II receptor blockers and longitudinal change in percent emphysema on computed tomography. The multi-ethnic study of atherosclerosis lung study, Ann Am Thorac Soc 14 (2017) 649-658.

[49] K.E. Kristensen, C. Torp-Pedersen, G.H. Gislason, M. Egfjord, H.B. Rasmussen, P.R. Hansen, Angiotensin-converting enzyme inhibitors and angiotensin II receptor blockers in patients with abdominal aortic aneurysms: nation-wide cohort study, Arterioscler. Thromb. Vasc. Biol. 35 (2015) 733-740. 